\title{
CONSTRUCTION OF THE MEMBERSHIP FUNCTION OF NORMAL FUZZY NUMBERS
}

\section{Anamika Dutta}

Department of Statistics, Gauhati University,

Guwahati-781014, Assam, India.

anamika.dut268@gmail.com

\author{
Dhruba Das \\ Department of Statistics, Gauhati University, \\ Guwahati-781014, Assam, India. \\ Dhrubadas16@gmail.com \\ Hemanta K. Baruah* \\ Department of Statistics, Gauhati University, \\ Guwahati-781014, Assam, India. \\ hemanta_bh@yahoo.com
}

\section{*Corresponding Author}

\begin{abstract}
A fuzzy real number $[\alpha, \beta, \gamma]$ is an interval around the real number $\beta$ with the elements in the interval being partially present. Partial presence of an element in a fuzzy set is defined by the name membership function. According to the Randomness- Fuzziness Consistency Principle, two independent laws of randomness in $[\alpha, \beta]$ and $[\beta, \gamma]$ are necessary and sufficient to define a normal fuzzy number $[\alpha, \beta$, $\gamma]$. In this article, we have shown how to construct normal fuzzy number using daily temperature data.
\end{abstract}

Keywords: Superimposition of Sets, Glivenko-Cantelli Theorem, Empirical Distribution Function, The RandomnessFuzziness Consistency Principle.

\section{Introduction}

Construction of normal fuzzy number has been discussed in [Baruah, 2011b, 2012] based on the Randomness - Fuzziness Consistency Principle deduced by Baruah [Baruah, 2010, 2011a, 2011b, 2011c, 2012]. In this article we shall show how to construct normal fuzzy numbers (Das et al, 2013a, 2013b) using the data of minimum and maximum temperature in Guwahati city for the month of December 2012 and up to $30^{\text {th }}$ January 2013. Partial presence of an element in a set is expressed in terms of the fuzzy membership function. But how exactly to construct the membership function of a fuzzy number mathematically remained a problem. Baruah (2010, 2011a, 2011b, 2011c, 2012) has shown that two laws of randomness are necessary as well as sufficient to define a normal law of fuzziness. This has led to a proper measure theoretic explanation of partial presence, and construction of fuzzy numbers can therefore be based on that.

We need to understand that if a variable $\mathrm{X}$ can assume values in an interval $[\mathrm{L}, \mathrm{U}]$ where $\mathrm{L}$ follows a law of randomness in the interval $[\alpha, \beta]$ while $U$ follows another law of randomness in the interval $[\beta, \gamma]$, then we are in a situation defining fuzzy uncertainty, with randomness defined in the measure theoretic sense (2011a). In such a case, Baruah's principle of consistency between randomness and fuzziness states that the distribution function of $\mathrm{L}$, which is known as the left reference function also with reference to fuzziness, in the interval $[\alpha, \beta]$ together with the complementary distribution function of $U$ which is known as the right reference function also in the interval $[\beta, \gamma]$, would give us the membership function of a normal fuzzy number $[\alpha, \beta, \gamma]$. The two concerned laws of randomness may or may not be geared to laws of probability because 
measure theoretically speaking the notion of probability need not actually appear in the definition of randomness in the sense that a probabilistic variable is necessarily random while a random variable need not be probabilistic (2011c). In what follows, we are going to explain how exactly a fuzzy number originates. We are going to show how exactly to construct a fuzzy number. We shall not assume anything heuristic in principle. It should be noted here that the temperature of a particular place at a particular time everyday is necessarily probabilistic, but the daily temperature in the same place is fuzzy.

\section{Methodology}

We collected daily temperature data in the city of Guwahati, India for the month of December 2012 and up to $30^{\text {th }}$ January 2013 from the daily newspaper The Assam Tribune ${ }^{34}$. We collected the values taken by $\mathrm{L}$ and $\mathrm{U}$ on those 61 days. These values were say $\left(\mathrm{a}_{1}, \mathrm{a}_{2}, \mathrm{a}_{3}, \mathrm{a}_{4}, \ldots \ldots, \mathrm{a}_{61}\right)$ and $\left(b_{1}, b_{2}, b_{3}, b_{4}, \ldots \ldots ., b_{61}\right)$ respectively.

Now, using the operation of set superimposition defined by Baruah (2010, 2011a, 2011 b, 2011c, 2012) we may proceed to construct normal fuzzy numbers as discussed in (Das et al, 2013a, 2013b), which would define the uncertainty associated with temperature variations. Here, the data should must satisfy the condition max $\left(a_{i}\right) \leq \min \left(b_{i}\right)$.

Suppose $\mathrm{a}_{(1)}, \mathrm{a}_{(2)}, \ldots, \mathrm{a}_{(61)}$ are values of $\mathrm{a}_{1}$, $\mathrm{a}_{2}, \ldots, \mathrm{a}_{61}$ arranged in increasing order of magnitude,

And $b_{(1)}, b_{(2)}, \ldots, b_{(61)}$ are values of $b_{1}, b_{2}$, $\ldots, b_{61}$ arranged in increasing order of magnitude.

We shall now superimpose the 61 intervals thus found, and shall normalize the frequency of occurrences by dividing by the total frequency.

Superimposing the intervals $\left[a_{1}, b_{1}\right],\left[a_{2}\right.$, $\left.\mathrm{b}_{2}\right], \ldots,\left[\mathrm{a}_{61}, \mathrm{~b}_{61}\right]$ and thereafter normalizing in the aforesaid manner is equivalent to superimposing

\footnotetext{
${ }^{34}$ The Assam Tribune, Guwahati, India, www.assamtribune.com.
}

the intervals $\left[a_{1}, b_{1}\right]^{(1 / 61)},\left[a_{2}, b_{2}\right]^{(1 / 61)}, \ldots,\left[a_{61}\right.$, $b_{61}{ }^{(1 / 61)}$ with constant level of partial presence $1 / 61$ for every interval. We shall thus get, subject to the condition that $\left[a_{1}, b_{1}\right] \cap\left[a_{2}, b_{2}\right] \cap\left[a_{3}, b_{3}\right]$ $\cap \ldots \ldots \ldots \cap\left[a_{61}, b_{61}\right]$ is not void, $\left[a_{1}, b_{1}\right]^{(1 / 61)}(S)$ $\left[\mathrm{a}_{2}, \mathrm{~b}_{2}\right]^{(1 / 61)}(\mathrm{S})\left[\mathrm{a}_{3}, \mathrm{~b}_{3}\right]^{(1 / 61)}(\mathrm{S}) \ldots \ldots \ldots . .(\mathrm{S})\left[\mathrm{a}_{61}\right.$, $\left.\mathrm{b}_{61}\right]^{(1 / 61)}=\left[\mathrm{a}_{(1)}, \mathrm{a}_{(2)}\right]^{(1 / 61)} \cup\left[\mathrm{a}_{(2)}, \mathrm{a}_{(3)}{ }^{(2 / 61)} \cup\left[\mathrm{a}_{(3)}\right.\right.$, $\left.a_{(4)}\right]^{(3 / 61)} \cup \ldots \ldots . . . \cup\left[a_{(61)}, b_{(1)}{ }^{(1)} \cup\left[b_{(1)}, b_{(2)}{ }^{(60 / 61)} \cup\right.\right.$ $\left[b_{(2)}, b_{(3)}\right]^{(59 / 61)} \cup \ldots \ldots \ldots \cup\left[b_{(60)}, b_{(61)}\right]^{(1 / 61),}$

where for example, $\left[b_{(1)}, b_{(2)}\right]^{(60 / 61)}$ represents the interval $\left[b_{(1)}, b_{(2)}\right]$ with level of partial presence of every element being 60/61 for every element in the interval.

We shall now proceed to construct the membership function of daily temperature data collected in the Guwahati city.

\section{Construction of the membership curve for the temperature of the Guwahati city}

We have collected data for the period of 61 days. The minimum temperature in increasing order of magnitude (in Celsius) were -

5.6, 5.7, 6, 6.5, 7, 7.6, 8.1, 8.2, 8.3, 8.5, 8.6, 8.7, $8.8,8.9,9,9.1,9.2,9.3,9.4,9.5,9.6,9.7,9.8,9.9$, $10,10.1,10.2,10.3,10.4,10.5,10.6,10.7,10.8$, $10.9,11,11.1,11.2,11.3,11.4,11.5,11.6,11.7$, $11.8,11.9,12,12.5,13.1,13.4,13.6,13.8,14$, $14.1,14.8,15.1,15.2,15.3,15.4,15.5,15.6,15.7$, 15.8

and similarly the maximum temperature in increasing order of magnitude (in Celsius) were-

$15.8,17.8,19.5,20.2,21.9,22.2,22.3,22.4,22.6$, $22.7,23,23.1,23.2,23.4,23.5,23.6,24,24.1$, 24.2, 24.3, 24.2, 24.3, 24.4, 24.5, 24.6, 24.8, 24.9, $25,25.1,25.2,25.3,25.4,25.6,25.7,25.9,26$, $26.1,26.2,26.3,26.4,26.5,26.7,26.8,26.9,27$, $27.1,27.2,27.3,27.4,27.5,27.6,27.7,27.8,27.9$, $28,28.1,28.2,28.3,28.4,28.5,28.6$.

We then superimposed the intervals making them equally fuzzy with constant level of partial presence equal to $1 / 61$ in every case. 
At this point, we would like to define what is known as an empirical distribution function in the statistical literature (Gibbons and Chakraborti, 1992, page- 25). An empirical distribution function may be considered as an estimate of the cumulative distribution function defining the randomness concerned. For a sample of size $\mathrm{n}$, this function $S_{n}(x)$, is defined as the proportion of values that do not exceed $X$. Accordingly, if $X_{(1)}, X_{(2)}, \ldots, X_{(n)}$ denote the order statistics of a random sample, its empirical distribution function would be given by

$$
\begin{aligned}
& S_{n}(x)=0, \quad \text { if } x<X_{(1)}, \\
& =k / n \text {, if } X_{(k)} \leq x<X_{(k+1)} \text {, } \\
& k=1,2, \ldots,(n-1), \\
& =1, \quad \text { if } x \geq X_{(n)}
\end{aligned}
$$

$\mathrm{X}$ here being random, so would be $\mathrm{S}_{\mathrm{n}}(\mathrm{X})$. Writing

we see that

$$
\begin{aligned}
\Delta_{i}(t) & =0, \text { if } X_{i}>t, \\
& =1, \text { otherwise. }
\end{aligned}
$$

$$
S_{n}(x)=\Sigma \Delta_{i}(x) / n .
$$

Therefore $n S_{n}(x)$ will have the law followed by the sum of $\mathrm{n}$ independent Bernoulli random variables $\Delta_{i}(x)$. Indeed in such a case, we would have

$$
\begin{aligned}
& \operatorname{Prob}\left[S_{n}(x)=k / n\right] \\
= & { }^{n} C_{k}\left[F_{x}(x)\right]^{k}\left[1-F_{x}(x)\right]^{n-k}
\end{aligned}
$$

for $\mathrm{k}=0,1, \ldots, \mathrm{n}$. Hence the mathematical expectation of $S_{n}(x)$ would be given by

$$
E\left[S_{n}(x)\right]=F_{x}(x) .
$$

Therefore, $S_{n}(x)$ converges uniformly to $F_{x}(x)$ almost surely. This leads to the Glivenko Cantelli theorem that states that the limiting value of the supremum of the difference between $S_{n}(x)$ and $F_{x}(x)$, as $\mathrm{n}$ becomes infinitely large, converges to zero almost surely.

After plotting the values (Fig.1), we have seen that the minimum temperature is an empirical distribution function of a random variable in the interval $[5.6,15.8]$, while the maximum temperature is a complementary empirical distribution function of another random variable in the interval $[15.8,28.6]$. Now, according to Baruah's randomness-fuzziness consistency principle, the theoretical distribution function of the minimum temperature in [5.6, 15.8] and the theoretical complementary distribution function of the maximum temperature in $[15.8,28.6]$ together define a normal fuzzy number $[5.6,15.8,28.6]$, which is clear from the diagram given below:

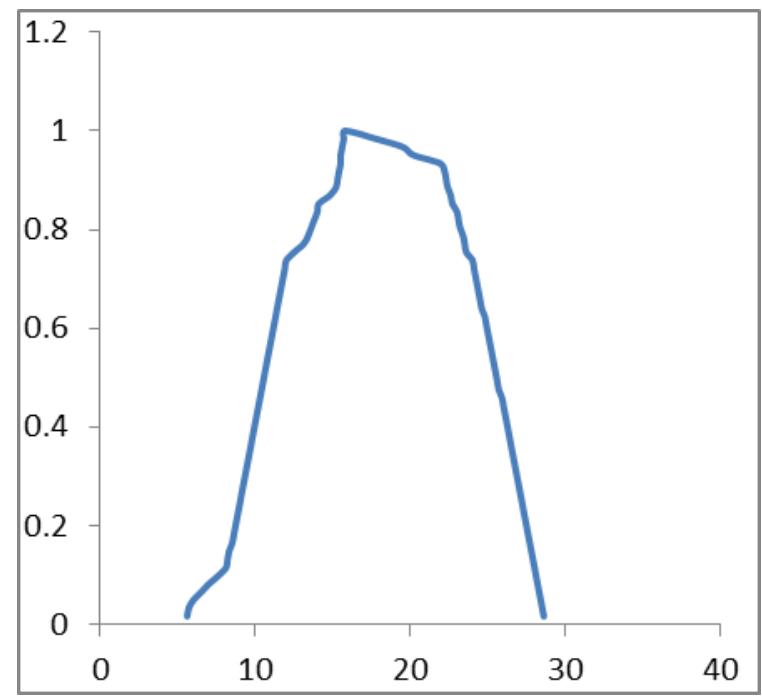

Fig. 1 Membership values of the temperature Thus, the fuzzy membership function in this example can be approximated as

$$
\mu_{\mathrm{x}}(\mathrm{x})=\left\{\begin{array}{lll}
0 & \text { if } & \mathrm{x}<5.6 \\
F(x) & \text { if } & 5.6 \leq \mathrm{x} \leq 15.8 \\
G(x) & \text { if } & 15.8 \leq \mathrm{x} \leq 28.6 \\
0 & \text { if } & \mathrm{x}>28.6
\end{array}\right.
$$

Here $F(x)$ and $G(x)$ are the Dubois - Prade left and right reference functions. $F(x)$ is a nondecreasing continuous function in the interval $[5.6,15.8]$ and $G(x)$ is a non-increasing continuous function in the interval $[15.8,28.6]$ i.e. $5.6 \leq \mathrm{x} \leq 15.8$ and $15.8 \leq \mathrm{x} \leq 28.6$ respectively.

From the diagram it is clear that the membership curve for the right reference function decreases with the increase in the temperature and that the left reference function increases with the increase in the temperature. 


\section{Fitting of the reference functions}

Taking the different values of temperature as an independent variable $X$ and the membership values as the dependent variable $Y$, we can fit the reference functions.

As for the right reference function, let us take a second degree polynomial as

$$
Y=a+b X+c X^{2}
$$

where $\mathrm{a}, \mathrm{b} \& \mathrm{c}$ are constants to be determined using the method of least squares for all $X>0$, also the maximum value of $Y$ cannot exceed unity and the lowest minimum value of $Y$ is 0 . Using the method of least squares the estimates of $\mathrm{a}, \mathrm{b}$ and $\mathrm{c}$ were computed.

We have found that the estimated membership values of the left reference function for the variables 15.8, 17.8 and 19.5 are $0.966438885, \quad 1.027201705$ and 1.016769375 respectively. We therefore had to do a little bit of adjustment in the sense that the above estimated membership values were approximated to unity, because the membership value always lies between 0 and 1. Similarly, we have also approximated the estimated membership value -0.009073307 as 0 . The equation thus found was

$Y=(-2.289355872)+0.3620051 X+$ $(-$ $0.00986975) X^{2}$

The curve concerned has been depicted in Fig.2 below:

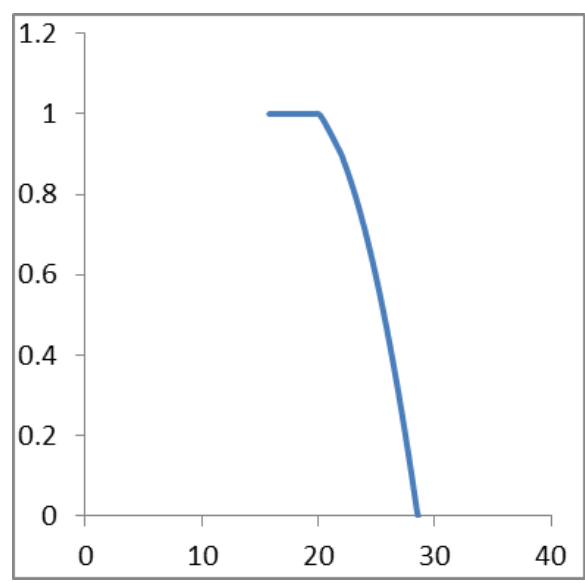

Figure 2: The right reference function
Similarly, the equation of the left reference function would be

$$
Y=a+b X+c X^{2}
$$

Where $\mathrm{a}, \mathrm{b} \& \mathrm{c}$ are constants to be determined using the method of least squares for all $X>0$. Using the method of least squares the estimates of $\mathrm{a}, \mathrm{b}$ and $\mathrm{c}$ were computed.

We have found that the estimated membership values of the left reference function for the variables 5.6, 5.7 and 6, are -0.098839495, $-0.086159926,-0.048348471$. We therefore had to do a little bit of adjustment in the sense that the above estimated values were approximated to 0 , because that the membership value always lies between 0 and 1 . The equation thus found was

$$
Y=\underset{(-0.001893767) X^{2}}{(-0.869344395)}+0.148195256 X+
$$

The curve concerned has been depicted in Fig.3 below:

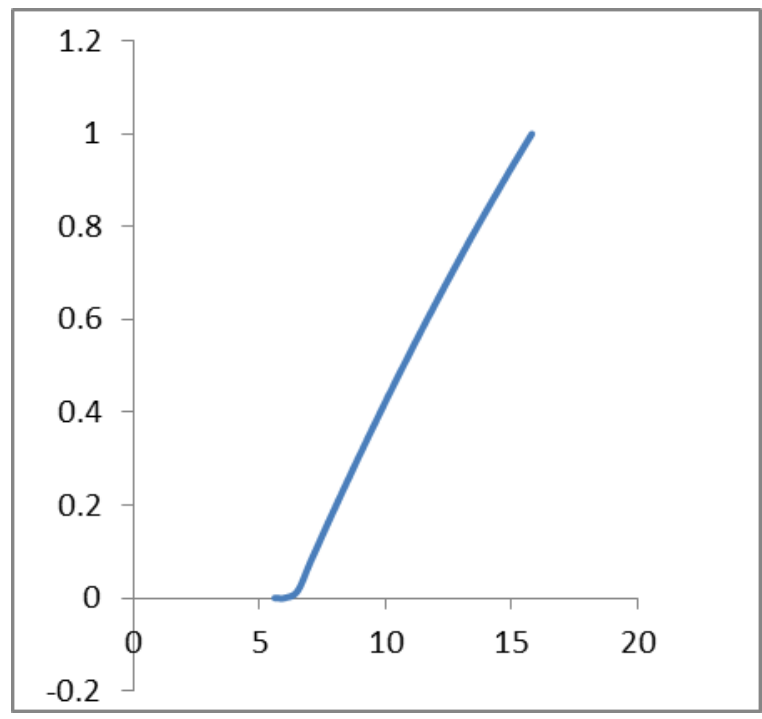

Figure 3: The left reference function

After combining the estimated left and right reference functions, we can construct the estimated membership curve (fig. 4), where the right reference function decreases from 1 to 0 and the left reference function increases from 0 to 1 . 
The curve concerned has been depicted in Fig.4 below:

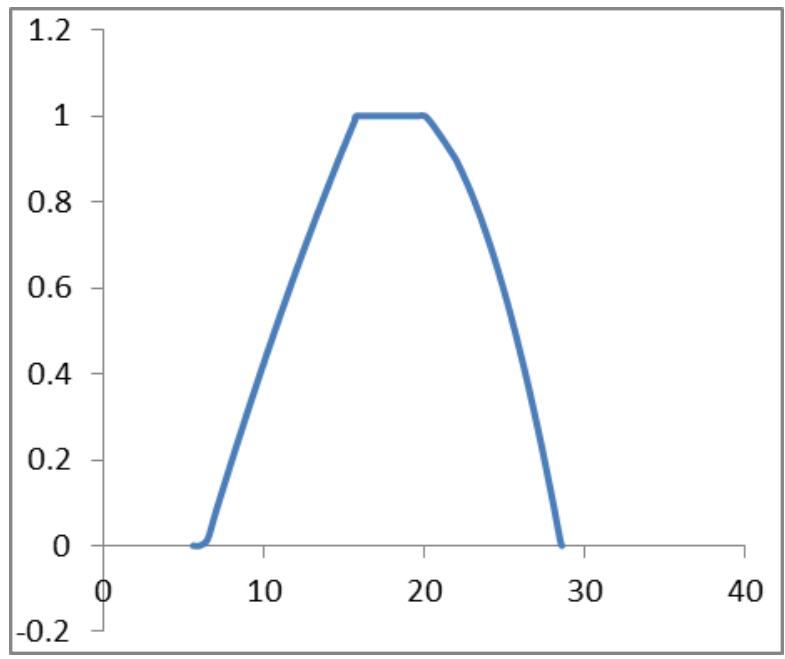

Figure 4: Estimated membership curve for temperature of Guwahati city.

\section{Conclusions}

In this article, from the daily temperature data of Guwahati city, we have been able to show how a normal fuzzy number can be constructed. We have seen that the two Dubois- Prade reference functions are to be defined on two probability spaces, the left reference function being a distribution function and the right reference function being a complementary distribution function. Indeed before using the concept of fuzziness in any situation, it should first be seen whether the theory of probability is sufficient to explain it. However, if the uncertainty concerned can be explained with reference to two laws of randomness, we need to apply the mathematics of fuzziness there.

\section{References}

1. Baruah, H. K. (2010), The RandomnessFuzziness Consistency Principle, International Journal of Energy, Journal
Information and Communications, Vol. 1, Issue 1, 37- 48.

2. Baruah, H. K. (2011a), In Search of the Root of Fuzziness: The Measure Theoretic Meaning of Partial Presence, Annals of Fuzzy Mathematics and Informatics, Vol. 2, No. 1, $57-68$.

3. Baruah, H. K. (2011b), Construction of the Membership Function of a Fuzzy Number, ICIC Express Letters, Vol. 5, Issue 2, 545-549.

4. Baruah, H. K. (2011c), The Theory of Fuzzy Sets: Beliefs and Realities, International Journal of Energy Information and Communications, Vol. 2, Issue 2, 2011, 1 - 22.

5. Baruah, H. K. (2012), Construction of Normal Fuzzy Numbers Using the Mathematics of Partial Presence, Journal of Modern Mathematics Frontier, Vol. 1, No. 1, 9- 15 .

6. Das, D., Mahanta S., Chutia, R. and Baruah, H. K. (2013a), Construction of normal fuzzy numbers: case studies with Indian stock exchange data, Annals of Fuzzy Mathematics and Informatics (in press).

7. Das, D., Dutta Anamika, Mahanta S., and Baruah, H. K. (2013b), Construction of normal fuzzy numbers: A case study with temperature data, Journal of Process Management - New Technologies, Vol. 1, Issue 1, 1- 6.

8. Gibbons J. D. and Chakraborti S., (1992); Nonparametric Statistical Inference, Third Edition, Marcel Dekker, Inc., New York. 